\title{
A NOTE ON INTEGRABLE REPRESENTATIONS
}

\author{
TERJE SUND
}

\begin{abstract}
It is shown that if all the coordinate functions of an induced representation $\pi=\operatorname{Ind}_{K}^{G}(\gamma)$ are integrable, then the representation $\gamma$ has the same property.
\end{abstract}

1. Let $G$ be a locally compact group. In the present note we are concerned with the behavior of integrable representations under the inducing process [4]. In [5] it was shown that if $\rho$ is an integrable cyclic representation of $K, K$ being a closed subgroup of $G$, then the induced representation $\operatorname{Ind}_{K}^{G}(\rho)$ is also integrable. As a partial converse to this we prove the following. If all the coordinate functions of $\operatorname{Ind}_{K}^{G}(\rho)$ are integrable then the same holds for $\rho$.

For simplicity we say that a continuous unitary representation $\pi$ of $G$ is completely integrable (c.i.) in case all of the coordinate functions $g \mapsto(\pi(g) v$, $v), g \in G, v \in H_{\pi}=$ the Hilbert space of $\pi$, are integrable w.r.t. left Haar measure on $G$. One may define completely square-integrable (c.s.i.) representation analogously. Note that irreducible integrable representations need not be completely integrable (see, e.g., Example 4). For square-integrable representations the corresponding holds if and only if the group is unimodular [2, Theorem 3].

2. It is known that square-integrable irreducible representations of ten can be obtained by inducing non-square-integrable representations (let, for instance, $G$ be the Heisenberg group mod a discrete central subgroup [5, 1.4]). The corresponding phenomenon can never occur for completely integrable representations, as the following result shows.

Theorem. Let $G$ be a separable locally compact group and $H$ a closed subgroup. Assume $G / H$ has a G-invariant measure, and let $\rho$ be a cyclic representation of $H$. Then we have:

(i) If $\rho$ is integrable then the cyclic representation $\operatorname{Ind}_{H}^{G}(\rho)$ is integrable.

(ii) If $\pi=\operatorname{Ind}_{H}^{G}(\rho)$ is completely integrable then $\rho$ is so.

Proof. (i) follows as (1.6) in [5] since the proof given there for unimodular groups can be adapted to this more general situation. Note only that the "cyclic" function $f$ in that proof can be chosen from $L^{1}(G)$ rather than from

Received by the editors July 31, 1975 and, in revised form, February 26, 1976.

AMS (MOS) subject classifications (1970). Primary 22D30.

Key words and phrases. Integrable representation, induced representation.

(1) American Mathematical Society 1976 
$C_{c}(G)$ (there is an inaccuracy at that point in [5]). Also, $f$ may be chosen so that $f\left(x^{-1}\right)=f(x)^{-}$; see $[3, \S 3]$. After these remarks the proof in [5] goes through.

To show (ii), assume that all the coordinate functions $x \mapsto(\pi(x) v, v)$ of $\pi$ are integrable. This is equivalent to the following: The linear functionals

$$
F_{v}: L^{\infty}(G) \cap L^{1}(G) \rightarrow \mathbf{C} ; \quad f \mapsto(\pi(f) v, v)
$$

are continuous in the sup-norm $\|\cdot\|_{G}$, all $v \in H_{\pi}$. That is, given $v \in H_{\pi}$ there is a constant $C_{v}$ such that

$$
\left|F_{v}(f)\right|=|(\pi(f) v, v)| \leqslant C_{v}\|f\|_{G}, \quad \text { all } f \in L^{\infty}(G) \cap L^{1}(G) ;
$$

see e.g. $[1,14.6 .1]$. Let $u \in H_{\rho}$ be arbitrary and put

$$
\varphi_{u}(t)=(\rho(t) u, u), \text { all } t \in C_{c}(H) .
$$

We wish to prove that $\varphi_{u}$ is a bounded linear functional on $C_{c}(H)$. Now, since $\pi=\operatorname{Ind}_{H}^{G}(\rho)$, we have from Blattner's theory of positive definite measures and induced representations that

$$
f \mapsto \mu(f)=\varphi_{u}\left(\left.f\right|_{H}\right) ; \quad f \in C_{c}(G),
$$

is a measure associated to the representation $\pi$. Hence we may assume that $\pi$ is constructed from $\mu$ in the usual way (see e.g. [5, the proof of (1.6)] for notation). By [5, Theorem 3.1] there is $f \in L^{1}(G)$ such that $[f]$ is a cyclic vector for $\pi$ and, in addition,

$$
\mu(\psi)=(\pi(\psi)[f],[f])_{\mu}, \quad \text { all } \psi \in C_{c}(G) .
$$

If $t \in C_{c}(H)$ let $\tilde{t}$ be any extension of $t$ to a continuous function with compact support on $G$ such that $\|\tilde{t}\|_{G}=\|t\|_{H}$ (Tietze's extension theorem). Then

$$
\begin{aligned}
\left|\varphi_{u}(t)\right| & =\left|\varphi_{u}\left(\left.\tilde{t}\right|_{H}\right)\right|=|\mu(\tilde{t})|=|(\pi(\tilde{t})[f],[f])| \leqslant C_{f}\|\tilde{t}\|_{G} \\
& =C_{f}\|t\|_{H}, \quad \text { all } t \in C_{c}(H),
\end{aligned}
$$

where $C_{f}$ is a constant depending only on $f$. Thus $\varphi_{u}$ is a bounded functional on $C_{c}(H)$. By the Riesz representation theorem there is a unique regular Borel measure $\nu$ on $H,\|\nu\|<\infty$, such that

$$
\int_{H} t(h)(\rho(h) u, u) d h=\varphi_{u}(t)=\int_{H} t(h) d \nu(h), \quad \text { all } t \in C_{c}(H) .
$$

Hence, by regularity of the measures involved we have $d \nu(h)=(\rho(h) u, u) d h$, and $\|\nu\|=\int_{H}|(\rho(h) u, u)| d h<\infty$. Since $u \in H_{\rho}$ was arbitrary, it follows that $\rho$ is completely integrable.

In view of the easy fact that every cyclic representation of a compact group is integrable, the following result is clear. 
COROLlary. Let $\pi$ be a representation of the separable group $G$ and assume that $\operatorname{Ind}_{K}^{G}(\rho)=\pi$ where $K$ is a compact subgroup of $G$ and $\rho$ is a cyclic representation of $K$. Then $\rho$ is integrable.

Good examples of completely integrable representations are super cuspidal representations of semisimple $p$-adic groups.

3. Using some Mackey theory one obtains, as a consequence of the above theorem,

Proposition. Let $G$ be a connected simply connected solvable Lie group, and suppose the nilradical is regularly embedded in $G$. Then $G$ has no completely integrable irreducible representations.

Sketch of Proof. Suppose $\pi \in \hat{G}$ is c.i., $\pi=\operatorname{Ind}_{H}^{G}(\rho)$ where $H$ is the stability group of some $\omega \in \hat{N}, N=$ nilradical of $G$. By the above theorem $\rho$ is c.i. Now $\omega$ is contained in the restriction $\left.\rho\right|_{N}$ as a subrepresentation. One may then verify as in [5, Lemma 1.1] that $\omega$ is integrable. This is a contradiction since simply connected nilpotent Lie groups have no integrable representations.

4. Finally we give an example showing that the converse of (ii) in the above theorem does not hold, even when $\rho$ and $\operatorname{Ind}_{H}^{G}(\rho)$ both are irreducible. Let $G$ be the semidirect product of the integers $Z$ and $T^{2}$ the direct product of two circles. $G=T^{2} \times_{\eta} Z$, where the integers act by

$$
\eta(n)\left(e^{2 \pi i a}, e^{2 \pi i b}\right)=\left(e^{2 \pi i(a+n b)}, e^{2 \pi i b}\right), \quad a, b \in \mathbf{R}, n \in Z .
$$

Letting $\chi\left(e^{2 \pi i a}, e^{2 \pi i b}\right)=e^{2 \pi i a}, a, b \in \mathbf{R}$, one may show that the stability group of $\chi$ under the action of $G$ by inner automorphisms equals $T^{2}$ so that $\operatorname{Ind}_{T^{2}}^{G}(\chi)=\pi$ is irreducible. To obtain a nonintegrable coordinate function for $\pi$, one may choose $f \in H_{\pi}$ as follows. Let for $t \in T^{2}, m \in Z, f(t m)$ $=\chi\left(m^{-1} t^{-1} m\right) /|m|$, for $m \neq 0$, and $f(t)=0$.

\section{REFERENCES}

1. J. Dixmier, Les $C^{*}$-algèbres et leurs représentations, Gauthier-Villars, Paris, 1964. MR 30 \#1404.

2. M. Duflo and C. Moore, On the regular representation of a nonunimodular locally compact group, J. Functional Analysis (to appear).

3. A. Hulanicki and T. Pytlik, On commutative approximate identities and cyclic vectors of induced representations, Studia Math. 48 (1973), 189-199. MR 49 \#3024.

4. G. Mackey, Unitary representations of group extensions. I, Acta Math. 99 (1958), 265-311. MR 20 \# 4789.

5. T. Sund, Square-integrable representations and the Mackey theory, Trans. Amer. Math. Soc. 194 (1974), 131-139. MR 49 \#9115.

Matematisk Institut, Universititet i Oslo, Blindern, Oslo 3, Norway 